\title{
Custodiol (Histidine-Tryptophan-Ketoglutarate) versus Crystalloid (Saint. Thomas) Cardioplegia on Myocardial Protection in Mitral Valve Replacement Surgeries
}

\author{
Amira Abo Elnasr Awad ${ }^{1 *}$ MD and Mohamed Attia Elkahely ${ }^{2}$ MD
}

\author{
* Corresponding Author: \\ Amira Abo Elnasr Awad \\ meronasr_2015@yahoo.com \\ Received for publication \\ February 16, 2020; accepted \\ July 7, 2020; published online \\ July 12, 2020.
}

Copyright 2020 The Authors published by Al-Azhar University, Faculty of Medicine, Cairo, Egypt. All rights reserved. This an openaccess article distributed under the legal terms, where it is permissible to download and share the work provided it is properly cited. The work cannot be changed in any way or used commercially.

doi:

1 *Department of Anesthesia and Intensive care, Faculty of Medicine for Girls, Al-Azhar University Cairo, Egypt.

${ }^{2}$ Department of Cardiothoracic Surgery, Faculty of Medicine for Girls, Al-Azhar University, Cairo, Egypt.

\begin{abstract}
Background: Cardioplegia is the solution used to arrest and protect myocardial function during cardiac surgery. Cardioplegia is widely used in open-heart surgery. Myocardial protection is a critical approach to reduce the myocardial complications that occur during and after cardiac surgery.

Aim of the study: This study was designed to assess the use of custodial (HTK) versus cold crystalloid cardioplegia (Saint-Thomas) on myocardial protection in mitral valve replacement surgeries.

Patient and methods: The study was done in the Cardiothoracic Department at Al-Zahraa University Hospital, Al-Azhar University, Cairo in the period from December 2016 to December 2019. A total of 50 patients undergoing mitral valve replacement surgeries (MVR) aged 25 to 60 years, ASA class II-III was randomly divided into two equal groups: Custodiol (HTK group, $\mathrm{n}=25$ ) or cold crystalloid cardioplegia (CCC group, $\mathrm{n}=25$ ) to produce cardiac arrest.

Result: No significant difference was identified intraoperatively in (ACC time, CPB time, duration of surgery) but there was a significantly higher number of return to a spontaneous rhythm in the custodiol compared to CCC_group (56\% versus $28 \% ; \mathrm{p}=0.045)$ and low volume in the HTK group $(1412.1 \pm 108.7$ vs. $1782.9 \pm 214.7 ; p=$ $0.0001)$. No significant differences between the two groups in CK-MB and troponin I preoperative or postoperatively, but there was a higher increase in their level at $24 \mathrm{~h}$ and $48 \mathrm{~h}$ postoperative in the CCC group versus HTK group. Duration of inotropic support postoperatively significantly higher in the CCC group.

Conclusions: The use of custodiol is effective than cold crystalloid cardioplegia for myocardial protection in mitral valve replacement surgeries

Keywords: Mitral valve surgeries; custodial; crystalloid cardioplegia; myocardial protection.

Authorship: All authors have a substantial contribution to the
article.
Disclosure: The authors have no financial interest to declare in
relation to the content of this article. The Article Processing
Charge was paid for by the authors.
\end{abstract}


decreasing ATP depletion thus improving the conditions of the heart at the end of surgery. On contrary cold cardioplegic solutions had economic cost and satisfactory clinical result and efficacy in a different group of patients and induced cardiac arrest by membrane depolarization but disadvantages compared with another cardioplegia it is known as poorly supplies oxygen, it also increases the percent of hemodilution which is already high after the priming volume and it also decreases the oncotic pressure. $^{5}$ Therefore we have done this study to compare the effects of custodiol and st.thomas cardioplegia on the myocardial protection during mitral valve replacement surgeries.

\section{PATIENT AND METHODS}

A prospective, randomized, comparative, doublecontrolled study was conducted on 50 adult patients in the Cardiothoracic Department in Al-Zahraa University Hospital, Al-Azhar University, Cairo from December 2016 to December 2019. All adult patients aged between 25 - 65 years, belonging to American Society of Anesthesiologists (ASA) classes II-III and undergoing mitral valve replacement surgery after taking approval of the local institutional ethical committee and informed consent obtained using computer-generated randomization (Random Allocation Software, M. Saghaei, Isfahan, Iran).

Patients were randomly assigned into two groups contains 25 patients (HTK group) received custodiol cardioplegia and (CCC group ) received cold crystalloid cardioplegia. Patients with a history of heart failure, severe cases of pulmonary hypertension, neurological manifestation, renal problems, hepatic insufficiency, and also patients suffering from pulmonary diseases, patients with redo cardiac surgery. Urgent or emergent operations and patients with ejection fraction (EF) less than $40 \%$ were excluded from the study.

Anesthesia Technique:

Pre-anesthetic check-up was done in all the patients one day before surgery and patients were assessed and required investigations were done including liver, and renal function tests, complete blood count, coagulation profile including \{prothrombin time, partial thromboplastin time and international normalizing ratio (INR) \}and prothrombin concentration and arterial blood gases were performed.

On arrival at the operating theatre, the peripheral intravenous line was inserted and all patients were premedicated with intravenous midazolam $0.01-0.1 \mathrm{mg} /$ $\mathrm{kg}$ and were monitored by Peripheral oxygen saturation(SpO2), , electrocardiogram (ECG ) and noninvasive blood pressure were monitored .Lastly patients connected to invasive blood pressure monitor (Infinity® Kappa - Drager) then induction of anesthesia was induced by thiopental in a dose of 0.5-2 $\mathrm{mg} / \mathrm{kg}$, IV fentanyl 3-5 $\mu \mathrm{g} / \mathrm{kg}$, and rocuronium bromide $(0.6-1.2 \mathrm{mg} / \mathrm{kg})$, as a muscle relaxant to facilitate intubation. Maintenance of anesthesia was achieved with a mixture of oxygen and air (1: 1), isoflurane at 1-2 $\%$ minimum alveolar concentration (MAC), rocuronium with infusion as $(5-10 \mu \mathrm{g} / \mathrm{kg} / \mathrm{min})$ and using fentanyl with a dosage of $0.05 \mu \mathrm{g} / \mathrm{kg} / \mathrm{min}$. Ventilation was adjusted to maintain an end-tidal carbon dioxide (EtCO2) value between 35 - $40 \mathrm{mmHg}$. Good monitoring of arterial blood pressure was obtained with the radial artery line as an invasive blood pressure monitoring. Also, it helps us for good monitoring of blood gases sampling all over the operation. A central venous catheter (central venous line) was placed under complete aseptic conditions ; urine output was monitor by insertion of a urinary catheter during operation. Also, core body temperature was assessed and monitored through the nasopharynx with its specific probe.

The surgical approach was done through a median sternotomy. The cardiopulmonary bypass (CPB) was established by an arterial cannula in the ascending aorta/aortic arch. Venous drainage was achieved through a two-stage cannula inserted in the right atrium or bicaval cannulation via the superior and inferior vena cava.

Before cannulation, a heparinization protocol of an initial bolus dose of 200-300 U per kilogram was given to maintain activated clotting time (ACT) between $400-450 \mathrm{sec})$ while we are placing the aortic pursestring sutures, $\mathrm{CPB}$ will be started following cannulation of the ascending aorta, superior vena cava, and inferior vena cava. We maintained all patients' body temperature at $\left(28^{\circ}-32^{\circ} \mathrm{C}\right)$ with good cooling. After aortic cross-clamping, the cardioplegia was administered in a short time to achieve the diastolic cardiac arrest. The solution was infused antegrade manner through aortic root cannula which inserted in the ascending aorta proximal to the cross-clamp, Topical cooling by ice slush was used in many patients to reach moderate hypothermia.

In Custodiol group (Bretschneider HTK, Bensheim, Germany at $2-8^{\circ} \mathrm{C}$ ) patients were given 25 $30 \mathrm{~mL} / \mathrm{Kg}$ in a single dose over 5-7 min, under an initial hydrostatic pressure of $80-100 \mathrm{mmHg}$. (maximum pressure $120 \mathrm{mmHg}$ ) another half dose was administered after three hours of cross-clamping or in case of any electrical or mechanical activity. In Cold crystalloid cardioplegia group (St. Thomas' solution at $4-8^{\circ} \mathrm{C}$ ) patients were given $20 \mathrm{~mL} / \mathrm{kg}$ in the first dose and followed by $10 \mathrm{~mL} / \mathrm{Kg}$ administered every 25-30 min at a pressure of 250$300 \mathrm{mmHg}$. Weaning from $\mathrm{CPB}$ started when the patients were rewarmed to $35-37^{\circ} \mathrm{C}$ followed by the removal of venous cannula and filtration. After that, protamine sulfate $(1-1.5 \mathrm{mg} / \mathrm{kg})$ was given to reverse the effects of heparinization and regain normal ACT just before cross-clamp removal and at the end of the CPB. Packed red blood cell transfusion and fresh frozen plasma were used when hematocrit levels were less than $22 \%$ during the intraoperative period. The operative parameters: Cardiopulmonary bypass time (CPB), Aortic cross-clamp time (ACC), the volume of cardioplegia, spontaneous rhythm restoration post $\mathrm{x}$ clamp, use of inotropic support during surgery, temporary pacemaker and duration of surgery.

Laboratory parameters: Serum CK-MB (MB fraction of creatine phosphokinase ) \& troponin I and serum sodium.

N.B Criteria for diagnosing a myocardial infarction: by at least two of the following:

Cardiac enzyme elevation (CK-MB $>30 \mathrm{U} / 1$ or troponin $\mathrm{I}>10 \mathrm{ng} / \mathrm{ml}$ ). 
The appearance of a new $\mathrm{Q}$ wave after suegery on the electrocardiogram of more than 0.03 second at least two serial electrocardiograms (ECG changes may be blurred by new-onset conduction disturbances, which common in the early postoperative period).

A new hypokinetic or akinetic area in the left or right ventricle by echocardiography.

Postoperative Parameters: Duration of mechanically ventilation, duration of inotropic support, ICU stay, and hospital stay.

Postoperative Complications: Sepsis, CVA (cerebrovascular accident), the incidence of renal dysfunction (elevated serum creatinine $>2 \mathrm{mg} / \mathrm{dl}$ or increase the baseline preoperative value by twice), respiratory failure, and mortality within 30 days.

The primary outcome: To detect the number of patients' needs for inotropic support and its duration. The secondary outcomes: Assessment of the serum level of CK-MB or troponin I, I.C.U stay postoperatively and postoperative complications in the HTK group versus CCC group.

Sample size justification :

A sample size calculation was performed depend on MedCalc® version 12.3.0.0 program "Ostend, Belgium", and calculation of statistical calculator based on $95 \%$ confidence interval and power of the study $80 \%$ with $\alpha$ error 5\%, According to a previous study, showed that the inotropic support need in group A (100\%), group B (65\%) vs. group C $(90 \%)$, was a statistically significant difference. So it can depend on this assumption of study ${ }^{7}$.the sample size was calculated according to these values used a minimal sample size of 57 patients was enough to find such a difference. Assuming a drop-out ratio of 5\%, $\mathrm{t}$

Sample size justification : A sample size calculation was performed depend on MedCalc $®$ version 12.3.0.0 program "Ostend, Belgium", and calculation of statistical calculator based on $95 \%$ confidence interval and power of the study $80 \%$ with $\alpha$ error 5\%, According to a previous study, showed that the inotropic support need in group A (100\%), group B (65\%) vs. group C (90\%), was a statistically significant difference. So it can be depend on this assumption of the study ${ }^{7}$.the sample size was calculated according to these values used a minimal samples size of 57 patients were enough to find such a difference. Assuming a drop-out ratio of $5 \%$. The sample size will be 50 patients; 25 patients in each group to produce cardiac arrest.

Statistical Analysis:

The statistical analysis was performed using a standard SPSS software package, IBM SPSS Statistics for Windows, Version 23.0. Armonk, New York: IBM Corp). Data were presented in the form of ( n. ) and (\%) for qualitative data and compared by using the chisquare test. The quantitative data were presented as mean values $\pm \mathrm{SD}$, numbers, and compared between two independent groups by using Independent t-test. The confidence interval was set to $95 \%$ and the margin of error accepted was set to $5 \%$.

\section{RESULTS}

There was no statistically significant difference between the CCC group and HTK group as regards demographic variables (age, sex, ASA, weight, height ) (table 2).

The duration of $\mathrm{CPB}$, aortic cross-clamping time (ACC), and the surgical duration, (P-value > 0.05) were comparable between both groups with no significant difference. But there was a statistically significant difference between the two groups as regarding the incidence of spontaneous rhythm restoration (56\%) in HTK group compared to (28\%) in CCC group and also in the use of inotropic support with the highest percentage (92\%) in group CCC and lowest percentage $(64 \%)$ in group HTK . The mean volume of transfusions for patients was highly significantly reduced in the HTK group versus the CCC group $(1412.1 \pm 108.7$ vs. $1782.9 \pm 214.7)$. Temporary pacing was used in (three patients) of the CCC group in comparison to (one patient) in the HTK group which was not statistically significant between both groups

(table 3).

There were no statistical differences between the two groups regarding $\mathrm{CK}-\mathrm{MB}$ and troponin (I) preoperative or at $24 \mathrm{~h}$ or $48 \mathrm{~h}$ postoperatively, but there was a higher increase in their release at $24 \mathrm{~h}$ and $48 \mathrm{~h}$ postoperative in CCC group in comparison to HTK group.

There was no statistical difference regarding serum sodium between both groups preoperative or postoperatively but showed a significant reduction in serum sodium significantly after clamping in the HTK group (table 4).

Duration of inotropic support significantly higher in CCC compared with HTK but other postoperative parameters were comparable in both groups (duration of ventilator stay, ICU stay, and hospital stay). (Figure 1)

The incidence of complications after surgery was comparable in both groups (table 5). 


\begin{tabular}{|c|c|c|}
\hline Components & $\begin{array}{l}\text { St. Thomas cardioplegia } \\
\text { (cold crystalloid cardioplegia) }\end{array}$ & $\begin{array}{c}\text { Custodiol cardioplegia } \\
\text { (HTK) }\end{array}$ \\
\hline $\mathrm{Na}+(\mathbf{m m o l} / \mathrm{L})$ & 144 & 15 \\
\hline $\mathbf{K}+(\mathbf{m m o l} / \mathbf{L})$ & 20 & 9 \\
\hline $\mathrm{Mg}++(\mathbf{m m o l} / \mathrm{L})$ & $16-32$ & 4 \\
\hline $\mathrm{Ca}++(\mathrm{mmol} / \mathrm{L})$ & $2.2-4.8$ & 0.015 \\
\hline Histidine (mmol/L) & - & 180 \\
\hline Tryptophan (mmol/L) & - & 2 \\
\hline Ketoglutarate (mmol/L) & - & 1 \\
\hline Mannitol (mmol/L) & - & 30 \\
\hline Dextrose (g/L) & 10 & - \\
\hline $\mathrm{NaHCO3}(\mathrm{mmol} / \mathrm{L})$ & 22.5 & - \\
\hline Osmolality (mosm/L) & $300-320$ & 300 \\
\hline $\mathbf{P H} \rightarrow$ & 5.5 & $7.02-7.20$ \\
\hline
\end{tabular}

Table 1: Composition of the cardioplegic solution [Viana et al. ${ }^{(7)}$ ].

\begin{tabular}{|l|c|c|c|c|c|}
\hline \multicolumn{1}{|c|}{ Parameter } & $\begin{array}{c}\text { CCC group } \\
(\mathbf{n = 2 5})\end{array}$ & $\begin{array}{c}\text { HTK group } \\
(\mathbf{n = 2 5})\end{array}$ & Test value & P value & Sig. \\
\hline Age $(\mathrm{yr})$ & $38.06 \pm 6.22$ & $39.03 \pm 9.61$ & $0.424^{\bullet}$ & 0.674 & NS \\
\hline $\begin{array}{l}\text { Sex } \\
\text { Male } \\
\text { Female }\end{array}$ & $\begin{array}{l}11(44.0 \%) \\
14(56.0 \%)\end{array}$ & $\begin{array}{l}12(48.0 \%) \\
13(52.0 \%)\end{array}$ & $0.081^{*}$ & 0.776 & NS \\
\hline $\begin{array}{l}\text { ASA class } \\
\text { II } \\
\text { III }\end{array}$ & $\begin{array}{l}13(52.0 \%) \\
12(48.0 \%)\end{array}$ & $\begin{array}{l}14(56.0 \%) \\
11(44.0 \%)\end{array}$ & $0.081^{*}$ & 0.776 & NS \\
\hline Weight(kg) & $72.11 \pm 5.51$ & $70.50 \pm 6.47$ & $-0.947^{\bullet}$ & 0.348 & NS \\
\hline Height(cm) & $165.65 \pm 6.57$ & $166.86 \pm 7.12$ & $0.624^{\bullet}$ & 0.535 & NS \\
\hline
\end{tabular}

Table 2: Demographic data of both groups.

Data were presented in the form of mean \pm SD or numbers and (percentages)

- Independent t-test; *: Chi-square test

Histidine Tryptophan Ketoglutarate (HTK); Cold Crystalloid Cardioplegia (CCC) 


\begin{tabular}{|l|c|c|c|c|c|}
\hline \multicolumn{1}{|c|}{ Parameter } & $\begin{array}{c}\text { CCC group } \\
(\mathbf{n = 2 5})\end{array}$ & $\begin{array}{c}\text { HTK group } \\
(\mathbf{n = 2 5 )}\end{array}$ & Test value & P-value & Sig. \\
\hline Aortic cross clamp time (min) & $85.4 \pm 30.2$ & $80.2 \pm 37.0$ & $-0.544^{\bullet}$ & 0.589 & NS \\
\hline Cardiopulmonary by pass time (min) & $175.3 \pm 28.3$ & $170.5 \pm 33.3$ & $-0.549^{\bullet}$ & 0.585 & NS \\
\hline Volume of cardioplegia (ml) & $1782.9 \pm 214.7$ & $1412.1 \pm 108.7$ & $-7.704^{\bullet}$ & 0.000 & HS \\
\hline $\begin{array}{l}\text { Spontaneous rhythm restoration post clamp } \\
\text { removal (no.) }\end{array}$ & $7(28.0 \%)$ & $14(56.0 \%)$ & $4.023^{*}$ & 0.045 & S \\
\hline Use Inotropic Support during surgery (no.) & $23(92.0 \%)$ & $16(64.0 \%)$ & $5.711^{*}$ & 0.017 & S \\
\hline Temporary pacemaker (no.) & $3(12.0 \%)$ & $1(4.0 \%)$ & 1.087 & 0.297 & NS \\
\hline Duration of surgery (min) & $250.3 \pm 20.0$ & $245.5 \pm 23.0$ & $-0.787^{\bullet}$ & 0.435 & NS \\
\hline
\end{tabular}

Table 3: Intraoperative data of both groups.

Data were presented in the form of mean \pm SD or numbers and (percentages)

- Independent t-test; *: Chi-square test. HTK: Histidine-Tryptophan-Ketoglutarate; CCC: Cold Crystalloid Cardioplegia.

\begin{tabular}{|c|c|c|c|c|c|}
\hline & $\begin{array}{c}\text { CCP group } \\
(\mathrm{n}=25)\end{array}$ & $\begin{array}{c}\text { HTK group } \\
(\mathrm{n}=25)\end{array}$ & Test value ${ }^{\bullet}$ & P-value & Sig. \\
\hline CK-MB (ng/mL ) & & & 0570 & 0566 & $N S$ \\
\hline CK-MB before operation & $1.45 \pm 1.31$ & $1.26 \pm 0.99$ & -0.579 & 0.500 & NS \\
\hline CK-MB $24 \mathrm{hr}$ postoperative & $15.6 \pm 3.3$ & $14.3 \pm 2.15$ & 1.650 & 0.105 & NS \\
\hline CK-MB $48 \mathrm{hr}$ postoperative & $7.48 \pm 30.1$ & $6.36 \pm 20.7$ & -0.153 & 0.879 & NS \\
\hline $\begin{array}{l}\text { Troponin I (cTnI) }(\mathbf{n g} / \mathbf{m L}) \\
\text { Troponin I before operation }\end{array}$ & $0.09 \pm 0.68$ & $0.03 \pm 0.29$ & -0.406 & 0.687 & NS \\
\hline Troponin I $24 \mathrm{hr}$ postoperative & $7.20 \pm 1.15$ & $6.81 \pm 0.48$ & -1.565 & 0.124 & NS \\
\hline Troponin I $48 \mathrm{hr}$ postoperative & $3.81 \pm 1.26$ & $3.35 \pm 0.57$ & -1.663 & 0.103 & NS \\
\hline $\begin{array}{l}\text { Serum } \mathbf{~ N a}(\mathbf{m m o l} / \mathbf{L}) \\
\text { Serum } \mathrm{Na}(\mathrm{mmol} / \mathrm{L}) \text { preoperative }\end{array}$ & $136.0 \pm 3.3$ & $135.6 \pm 2.8$ & -0.462 & 0.646 & NS \\
\hline Serum $\mathrm{Na}(\mathrm{mmol} / \mathrm{L})$ after clamping & $131.0 \pm 5.4$ & $128.0 \pm 1.15$ & -2.717 & 0.009 & HS \\
\hline Serum $\mathrm{Na}(\mathrm{mmol} / \mathrm{L})$ after $\mathrm{CPB}$ & $129.5 \pm 4.9$ & $127.6 \pm 5.7$ & 1.264 & 0.212 & NS \\
\hline
\end{tabular}

Table 4: Laboratory parameters. Data were presented in the form of mean $\pm \mathrm{SD}$

- Independent t-test. CK-MB= MB fraction of creatine phosphokinase, $\mathrm{cTnI}=$ cardiac Troponin I

\begin{tabular}{|c|c|c|c|c|c|}
\hline Postoperative Complications & $\begin{array}{c}\text { CCC group } \\
(\mathbf{n = 2 5})\end{array}$ & HTK group (n=25) & Test value $^{*}$ & P-value & Sig. \\
\hline Sepsis & $1(4.0 \%)$ & $1(4.0 \%)$ & 0.000 & 1.000 & NS \\
\hline Cerebrovascular accident & $1(4.0 \%)$ & $(0 \%)$ & 1.020 & 0.313 & NS \\
\hline Acute kidney injury & $(0 \%)$ & $(0 \%)$ & NA & NA & NA \\
\hline Respiratory failure & $1(4.0 \%)$ & $(0 \%)$ & 1.020 & 0.313 & NS \\
\hline Mortality & $(0 \%)$ & $(0 \%)$ & NA & NA & NA \\
\hline
\end{tabular}

Table 5: Postoperative Complications. .Data were presented as numbers and percentages. ${ }^{*}$ Chi-square test. 


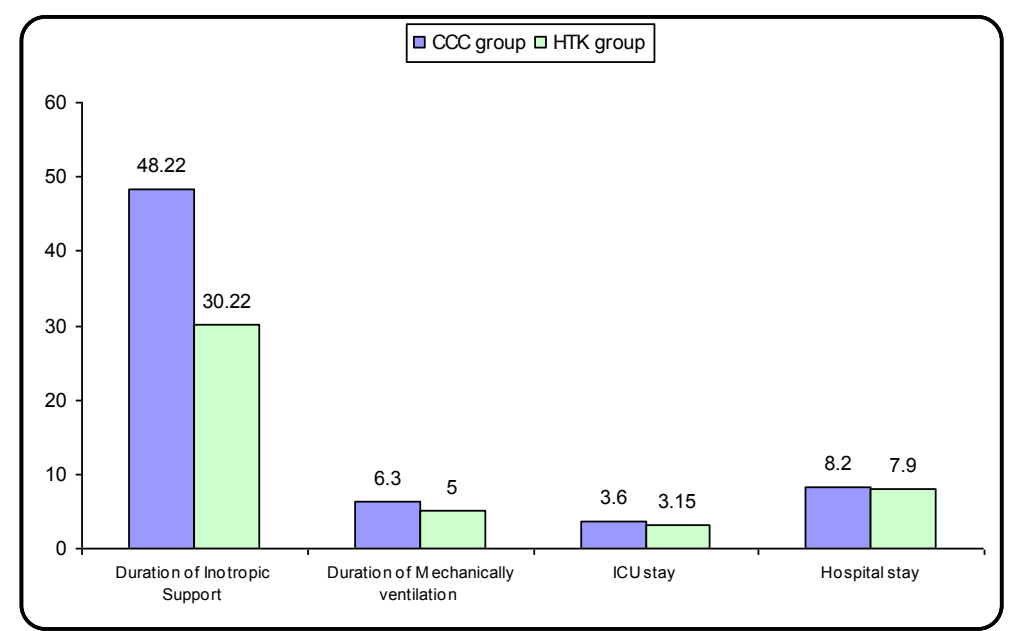

Fig. 1: Comparison between both groups regarding postoperative data.

\section{DISCUSSION}

During cardiac surgery, myocardial damage is induced by many factors such as ischemic injury, reperfusion injury, and the inflammatory response associated with cardiopulmonary bypass. Protective measures designed to attenuate ischemic injury, one of them is rapid ischemic arrest with a "cardioplegic", solution. ${ }^{8,9}$

The purpose of cardioplegia is to cause myocardial arrest, support normal physiology during ischemia, and to decrease the metabolism.

This study designed to compare the efficacy of a single dose of (custodiol) versus multiple doses of cold crystalloid cardioplegia on myocardial protection on patients with mitral valve replacement surgeries.

Few studies compared myocardial protection between HTK and St. Thomas cardioplegia $10,11,7$

In agreement with the current result ,study who compared HTK with St. Thomas cardioplegia for myocardial protection and found no significant differences observed between both groups in aortic cross-clamp or CPB times. The volume of transfusions was significantly reduced in the HTK group versus the CCC group (772.1 \pm 88.7 vs. $1352.9 \pm 214.7 \mathrm{ml}$ ) .The rates of spontaneous defibrillation in the HTK group were significantly higher than of CCC group (92.9 vs. $72.2 \%$ ), the changes in serum sodium observed in the HTK group decreased significantly after clamping. But the duration of ICU stay and postoperative hospitalization was significantly shorter in HTK than the CCC group (10.5 \pm 1.4 vs. $20.1 \pm 4.4$ days) also mortality in the HTK group was significantly lower than that of the CCC group (2.8 vs. $22.2 \%) .{ }^{10}$

Confirming our finding, study compared three solutions for cardioplegia in pediatric cardiac surgery: HTK solution (group A), Blood Cardioplegia (group B) and St. Thomas Cardioplegia (group C), there was no significant difference between three groups regarding duration of $\mathrm{CPB}$, aortic cross-clamping time and the surgical duration, but differ from our study result in a higher percentage of patients needed inotropic support after $\mathrm{CPB}$ was reported in the group (A) then group C however the lowest one was in the (group B). On the other hand, there was a significant difference between both groups regarding the time of mechanical ventilation, length of ICU stay with the highest mean level among (group B). ${ }^{7}$

Against the present study, literature who compared custodiol versus modified St. Thomas cardioplegia in coronary artery bypass grafting and found there were significant longer cross-clamp and cardiopulmonary bypass times in the custodiol compared versus St. Thomas cardioplegia $(49.1 \pm 19.0$ vs. $41.0 \pm 12.9$ minutes) and $(82.2 \pm 23.7$ vs. $74.5 \pm 18.5$ minutes $)$ respectively while spontaneous rhythm recovery rate was similar in both groups. the amount of cardioplegia was significantly larger in the custodiol vs. st.thomas group (1667 vs. $1306 \mathrm{ml}$ respectively), but this study similar to our result in inotropic support $(9.0 \%$ vs. $12.0 \%)$ and postoperative complications which were comparable between two groups but ICU and hospital stay were less frequent in the custodiol group, with no statistical significance $(14.8 \%$ vs. $24.0 \%, p=0.348)$ and $(7.4 \%$ vs. $12.0 \%$ respectively). ${ }^{11}$ Some of their results were different from ours maybe because of the differences in the type of surgery and a relatively high number of patients (104 patients ) have been included in their study.

Similar to the current result but used cold blood cardioplegia ,study who compared HTK versus cold blood cardioplegia in pediatric surgery. The inotropic dose needed and duration of its usage were significantly high in cold blood cardioplegia compared with HTK and spontaneous recovery of the heart was higher in the HTK group but CPB duration was insignificant between (HTK group) and ( cold blood cardioplegia group). ${ }^{12}$

Also, study who compared custodiol with cold blood cardioplegia, There was no significant difference in aortic cross-clamp time between the cold 
cardioplegia and HTK group (73 \pm 3 vs $75 \pm 3$ minutes; $\mathrm{P}=0$. 81). And also no difference in inotropic support but there was a significant difference as regarding spontaneous fibrillation $(71 \%)$ in patients receiving custodiol resumed cardiac activity compared to (13\%) of patients in the group receiving cold blood cardioplegia. ${ }^{13}$

In agreement with our study, literature measured serum sodium and osmolality after HTK cardioplegia at different Intra- and postoperative time-points, patients showed a significant reduction in serum sodium in HTK compared with other cardioplegia solution significantly after clamping. However, there was not any significant alteration in osmolality thus suggesting isotonic hyponatremia ${ }^{14,15,10}$. But in our study, we not measured serum osmolality.

The present study observed that custodiol group and cold crystalloid group as regarding CK-MB and troponin I post-surgery, there was no statistically significant difference between both groups. Supporting our result , study compared HES with St. Thomas cardioplegia during coronary artery bypass surgery to evaluate myocardial protection of both groups and found that there was no significant difference in the values of troponin I and CK-MB post-surgery. The peak values of all enzymes were $6 \mathrm{~h}$ after surgery. ${ }^{11}$

Similarly, a meta-analysis performed by ${ }^{16}$ (who studied the effects of blood cardioplegia versus crystalloid cardioplegia during cardiac surgery of pediatric) reported that cardiac troponin I level postoperatively was not significantly different between two groups with no evidence of improvement in myocardial injury for either groups .Also study comparing three groups ( custodiol group , st.thomas cardioplegia group and blood cardioplegia solution) during pediatric cardiac surgery and evaluate protective effects of three groups on the myocardial of the heart and found that CK-MB and troponin $t$ levels were not statistically significantly different among the three groups with no superiority for $\mathrm{HTK}^{7}$.

Also, study who reported that there was no significant difference between custodiol and crystalloid cardioplegia as regarding CK-MB levels and troponin I. ${ }^{17}$

Against our result,study who observed that creatine kinase-MB isoenzyme and troponin $t$ levels were significantly higher in group HTK compared with cold blood cardioplegia, the highest levels were found $6 \mathrm{~h}$ postoperatively. ${ }^{12}$ Their results were different from our study may be due to the use of cold blood cardioplegia not cold cardioplegia. The main limitations of the study, it was done in a single institution, the operations were performed by different surgeons and therefore, lack standardization. We didn't analyze postoperative ejection fraction and didn't use of transesophageal echocardiography.

\section{CONCLUSION}

HTK cardioplegia offers better myocardial protection compared to cold crystalloid cardioplegia detected by less need to inotropic support, low level of CK-MB and troponin-I postoperatively, less hospital, I.C.U stays and other parameters which may suggest that its benefits could be ascertained in a larger study.

\section{REFERENCES}

1. Mentzer RM. Myocardial protection in heart surgery. Journal of CardiovascularPharmacology and Therapeutics. 2011; 16(3-4):290-7.

2. Buckberg GD and Athanasuleas CL. Cardioplegia: solution or strategies? Eur J Cardiothorac Surg 2016; 50:787-91.

3. Turer AT and Hill JA. Pathogenesis of myocardial ischemia-reperfusion injury and rationale for therapy. Am J Cardiol 2010; 106(3):360-8.

4. Triana JR, Yanagihashi $M$, and Larson DF. Mathematical modeling of buffers used in myocardial preservation. Perfusion. 2007; 22(5):35362.

5. Bretschneider HJ. Myocardial protection. The Thoracic and Cardiovascular Surgeon. 1980; 28(5):295-302

6. Viana FF, Shi WY, Hayward PA, et al. Custodiol versus blood cardioplegia in complex cardiac operations: an Australian experience. Eur $J$ Cardiothoracic Surg 2013; 43: 526-32.

7. Hamed MA and Ghaffar AA. Comparative Study between Three Solutions for Cardioplegia in Pediatric Cardiac Surgery: Histidine-TryptophanKetoglutarate (HTK) Solution, Blood Cardioplegia, and Crystalloid (St. Thomas) Cardioplegia. J Anesth Clin Res 2018; 9(4):1-6.

8. Careaga G, Salazar D, Tellez S, et al. Clinical impact of histidine-ketoglutarate-tryptophan (HTK) cardioplegic solution on the perioperative period in open-heart surgery patients. Archives of Medical Research. 2001; 32(4):296-9.

9. David J Chambers. Mechanism of cardiac damage associated with cardiac surgery, Heart Metab. 2005; 29:5-9.

10. Lin YZ, Huang JB, Li XW, et al. Clinical comparative analysis of histidine-tryptophanketoglutarate solution and St. Thomas crystalloid cardioplegia: A 12-year study from a single institution. Exp Ther Med 2017; 14(3):2677-82.

11. Cvetkovic D, Kocica M, Soskic L, et al. Comparison of custodiol and modified st.thomas cardioplegia for myocardial protection in coronary artery bypass grafting: VSP, 2018 4:1-18.

12. El-Morsy GZ, Abdullah HM, Abo-Haded HM, et al. Does the type of cardioplegia affect the myocardial and cerebral outcome in pediatric open 
cardiac surgeries? Ain-Shams J Anaesthesiol, 2014; 7: 242-49.

13. Braathen B and Tonnessen T. Cold blood cardioplegia reduce the increase in cardiac enzyme levels compared with cold crystalloid cardioplegia in patients undergoing aortic valve replacement for isolated aortic stenosis. J Horac Cardiovasc Surg 2010; 139: 874-80.

14. Lindner G, Zapletal B, and Schwarz C. Acute hyponatremia after cardioplegia by histidinetryptophane-ketoglutarate--a retrospective study. $J$ Cardiothorac Surg 2012; 7:52.

15. Lueck S, Preusse CJ and Welz A. Clinical relevance of HTK induced hyponatremia. Ann Thorac Surg 2013; 95:1844-5.

16. Fang Y, Long C, Lou S, et al. Blood versus crystalloid cardioplegia for pediatric cardiac surgery: a meta-analysis perfusion 2014; 30: 529-36.

17. Kuslu S, Zeyneloglu P, Pirat A, et al. Comparison of the effects of histidine-tryptophan-ketoglutarate solution and crystalloid cardioplegia on myocardial protection during pediatric cardiac surgery. Critical Care 2014; 18: 178. 